DOI: https://doi.org/10.46296/yc.v5i9edespsep.0131

\title{
PYMES Y DESARROLLO ESTRATÉGICO EN EL ECUADOR
}

\section{SMES AND STRATEGIC DEVELOPMENT IN ECUADOR}

\author{
Zanella-Martínez Leonardo Mauricio ${ }^{1}$; Macías-Ugalde Lexandra Tatiana ${ }^{2}$; \\ Balseca-Macías Paola Estefanía ${ }^{3}$ \\ ${ }^{1}$ Investigador ecuatoriano independiente. Profesor universitario de instituciones de Educación \\ Superior. Consultor empresarial. Ecuador. Correo: leozanella80@gmail.com. ORCID ID: \\ https://orcid.org/0000-0002-2388-5767
}

${ }^{2}$ Profesora investigadora de la Universidad Laica Eloy Alfaro de Manabí. Ecuador, ULEAM. Manta, Ecuador. Correo: lexandra.macias@uleam.edu.ec. ORCID ID: https://orcid.org/00000001-7739-2581

${ }^{3}$ Profesora investigadora de la Universidad Laica Eloy Alfaro de Manabí. Ecuador, ULEAM. Manta, Ecuador. Correo: paola.balseca@uleam.edu.ec. ORCID ID: https://orcid.org/0000-00018412-1616

\section{Resumen}

La cultura organizacional ha sido un factor determinante en el éxito o fracaso en los procesos comerciales de cualquier organización. Numerosos son los estudios con referente a la cultura organizacional los cuales han evidenciado el efecto que tiene sobre el rendimiento de las actividades de las organizaciones. Ineludiblemente con el proceso del desarrollo estratégico se busca mantener un diálogo inmutable e indeleble con el consumidor, acorde con sus preferencias y necesidades. De este modo es como determinadas marcas se mantienen latentes tanto en la mente como en las elecciones de compra de los consumidores. Indiscutiblemente, el objetivo principal de esta tentativa radica en edificar el dinamismo con el consumidor, puesto que la responsabilidad de las empresas no se limita únicamente al proceso de venta. Dado que únicamente si se quiere crear una estructura sólida de consumidores recurrentes que aporten estabilidad a los ingresos de las empresas, resulta ineludible atender las necesidades de los consumidores.

Palabras clave: PYMES, desarrollo, cultura organizacional, empresa.

\begin{abstract}
Organizational culture has been a determining factor in the success or failure of any la cultorganizational culture which have shown the effect it has on the performance of the activities of the organizations. Inevitably, the strategic development process seeks to maintain an immutable and indelible dialogue with the consumer, in accordance with their preferences and needs. This is how certain brands remain latent both in the mind and in the purchasing choices of consumers. Unquestionably, the main objective of this attempt lies in building dynamism with the consumer, since the responsibility of companies is not limited only to the sales process. Since only if you want to create a solid structure of recurring consumers that provide stability to the income of companies, it is inescapable to meet the needs of consumers.
\end{abstract}

Keywords: SMEs, development, organizational culture, company.

Información del manuscrito:

Fecha de recepción: 29 de junio de 2021.

Fecha de aceptación: 09 de septiembre de 2021.

Fecha de publicación: 17 de septiembre de 2021. 


\section{Introducción}

En primera instancia, resulta de suma importancia contextualizar las definiciones etimológicas de desarrollo estratégico y comercial, para posteriormente agilizar el entendimiento del impacto del proceso de desarrollo estratégico en el marco del proceso comercial correspondiente a las pequeñas y medianas empresas (Pymes). En ese sentido, cabe destacar que el proceso comercial se refiere al proceso de la compra de un producto o servicio. Durante este período, las empresas tienen el deber de llevar a cabo una atención al consumidor dirigida a ejecutar estrategias que mejoren la experiencia del mismo después de una adquisición. Asimismo, resulta optimo tener en cuenta las problemáticas preexistentes, así también como depender del grado de desarrollo referente a determinada locación. A partir de la caracterización de las labores de responsabilidad social, y un sistema de vinculación en la sociedad, resulta de vital importancia desarrollar un marco viable $y$ medible para las pequeñas $y$ medianas empresas que desean no hacer de su responsabilidad una actividad humanística convertida enteramente en una estrategia de imagen corporativa. Concretamente, a través del desarrollo estratégico de las pequeñas y medianas empresas pueden lograr una integración y desarrollo comercial considerablemente benéfico a largo plazo.

\section{Perspectivas del desarrollo Estratégico y las Pymes}

Las pequeñas y medianas empresas (Pymes) no solo deben ser sostenibles, sino que debe generar beneficios para continuar con las actividades operacionales de la misma. En síntesis, además de ser posible, la sustentabilidad resulta absolutamente necesaria (Devoto, Liliana, 2019). En este marco teórico la intrínseca necesidad radica al poner marcha dos pilares fundamentales, en los cuales predomina la Responsabilidad Social Corporativa (RSC). Esto lo corroboran autores como González, A. G. (2014) quien afirma que la calidad de servicio y la satisfacción del cliente se han considerado tradicionalmente como las principales vías para incrementar el índice de fidelidad del consumidor. Este resulta ser del mismo modo el 
caso del servicio en el contexto de automoción, un sector comercial altamente rentable en países de primera línea como en el caso de España. En este sector comercial se dedica una cantidad significativa de recursos a la mejora de la calidad de servicio. Bajo esta premisa, Las pymes que se han internacionalizado se embarcan en la aventura de la internacionalización de diversas formas. A veces valientemente competitivos, por lo que saben cómo desarrollar estrategias eficientes para mantener posiciones sólidas en los mercados externos (Raymond, Louis \& St-Pierre, Josée, 2018). Tal es el caso de las empresas que han dado un salto para organizarse y desarrollar de manera efectiva sus especificidades. A esto le siguieron innovaciones radicales. Sin embargo, solo unos pocos estudios se han centrado en analizar el desarrollo internacional de los países emergentes. Así, es posible estudiar esta evolución en una etapa más temprana del valor agregado. De hecho, a veces algunas PYME exportadoras de los países en desarrollo son capaces de penetrar en el mercado exterior sin lograr el liderazgo mundial; ese es el caso de las empresas industriales, las cuales según Zribi, Hajer \& Zouaoui, Mahmoud. (2013) no están en la mejor posición en su área para crear y adoptar innovaciones radicales, entonces deciden optar por innovaciones micro-radicales.

\section{Pymes y desarrollo Estratégico en Latinoamérica}

A nivel de Latinoamérica, Indiscutiblemente la tecnología en la industria ha mejorado los procesos, bienes y experiencia del consumidor. Debido a esto, la importancia del desarrollo estratégico es una tendencia en aumento. Por ello, autores como Aslam, Wajeeha \& Farhat, Kashif. (2020) otorgan principal importancia a identificar el impacto de la entrega de calidad del servicio posventa en la satisfacción general de los clientes en el sector minorista. Además, el impacto de la satisfacción general en el boca a boca y la intención de recompra. En este contexto, la calidad de la entrega del servicio posventa afecta la satisfacción general del consumidor, lo que conduce a la intención de retorno, generando la lealtad de marca. Por ello, las pequeñas y medianas empresas (Pymes) deben implementar 
estrategias de marketing, puesto que esto les permite diferenciar sus ofertas para desarrollar la relación a largo plazo con sus consumidores, desencadenando tendencias de comercio dinámicas donde los clientes están muy involucrados.

Debido a la creciente globalización, el desarrollo tecnológico, así también como los cambios sociopolíticos y económicos, las pequeñas y medianas empresas alrededor del mundo se ven obligadas a involucrarse cada vez más en cuestiones de edificar la innovación en sus procesos de producción. Sin embargo, muchas veces se desconoce cuáles son los factores fundamentales que deben modificarse dentro de la estructura de la empresa con el propósito de elevar su competitividad en el mercado. (Ycaza, D. E. M., \& Sánchez-Riofrío, A. M, 2016). En este marco contextual, resulta optimo afirmar que las denominadas Pymes simbolizan a nivel global un aporte significativo para la economía de los países en que se desarrollan, sin embargo, se deben instaurar mecanismos para que las mismas puedan competir en el mercado frente a las grandes empresas, ya que tienen múltiples desventajas frente a empresas multinacionales de renombre. Pese a esto, autores como Guillermo, Vázquez \& Mejía Trejo, Juan \& Núñez-Moreno, Tania. (2016) afirman que las pymes son vulnerables a presentar diversas problemáticas que no les permiten alcanzar un desarrollo económico de manera óptima y acelerada, puesto que tal como indican, para lograrlo se requiere que estas constantemente implementen estrategias claves y exploren sus propias fortalezas dentro de sus estructuras internas, mismas que al ser analizadas, desencadenen en una búsqueda constante de planes estratégicos que ayuden a maximizarlas.

\section{Pymes y desarrollo Estratégico en el Ecuador}

A nivel de Ecuador, la gestión de las relaciones con los consumidores, actualmente constituye una herramienta primordial en el contexto de la toma de decisiones y en el desempeño de los objetivos y estrategias empresariales $y$ estratégicos (Mejia Zambrano, 2019). Indiscutiblemente, las tecnologías y los sistemas de 
información facilitan a las empresas mantener la ventaja competitiva en el mercado, y recíprocamente atender las necesidades y requerimiento de los consumidores. Por ello, resulta de vital importancia de los modelos de la gestión de las relaciones entre distribuidores y consumidores que han sido eficientes en varias empresas enfocadas en el ámbito de los servicios, de igual, resulta indispensable contextualizar a las pymes de los sectores enfocados en la venta de servicios, como en el caso oportuno de la climatización y su actual gestión de consumidores. En este marco contextual, la principal problemática radica en la mala administración y manejo de los consumidores, para lo cual resultaría optimo que las pymes adopten modelos destinados a mejorar sus relaciones con los consumidores con variables y dimensiones aplicables en múltiples contextos, donde la gestión de fidelización de clientes y el desarrollo estratégico puedan plantearse como las variables influyentes en este tipo de estrategias. En base a este análisis, resulta acertado afirmar que la cantidad de productos y servicios ofertados, así también como el respectivo seguimiento de los consumidores son los factores no aceptables dentro del modelo de mejora relacional, en el cual las pymes deben centrar sus esfuerzos para forjar una efectiva gestión de clientes. Esto lo corroboran autores como Morocho, F. R. A., \& Armas, C. S. B. (2017) quienes afirman que, en el sector automotor a nivel de Ecuador, se ha mantenido históricamente una participación considerable en la economía del país, esto debido principalmente a los ingresos que genera en todas las operaciones económicas directas e indirectas que involucra. En este marco contextual, los autores afirman que, a partir de la industria, y el sector económico automotriz se han impulsado otras industrias como la metalúrgica, siderúrgica, metalmecánica, petrolera, minera, petroquímica, entre otras industrias derivadas que resultan indispensables para la manufactura y construcción de vehículos automotores. El sector automotor compone a diferentes actores, como en el caso de las empresas autopartistas, y en específico a proveedoras de partes y piezas automotrices. por otra parte, las empresas ensambladoras de vehículos han sido los actores que 
impusieron en su momento los estándares productivos en la sucesión de producción de este sector.

\section{Conclusiones}

Hoy por hoy, existe una ardua competencia entre las empresas pertenecientes a esta industria con respecto obtener la atención del consumidor. En este contexto, la calidad en el servicio propiciado por las empresas es uno de los factores principales que deben ser implementados dentro de cada una de las empresas. En consecuencia, una eficaz y correcta gestión con respecto a la atención al consumidor resulta en la mejor estrategia que se puede implementar para lograr una empresa de éxito comercial, y de esa manera poder experimentar un desarrollo en períodos complicados como el panorama actual que atraviesan las pequeñas y medianas empresas del Ecuador en la época contemporánea.

\section{Bibliografía}

Aslam, Wajeeha \& Farhat, Kashif. (2020). Impact of after-sales service on consumer behavioural intentions.
International Journal of Business and Systems Research. 14.44. 10.1504/IJBSR.2020.104145.

Delgado, D., \& Chávez, G. (2018). Las Pymes en el Ecuador y sus fuentes de financiamiento. Revista Observatorio de la Economía Latinoamericana, 1-16.

Devoto, Liliana. (2019). La sustentabilidad en las pymes, ¿es posible? Cuadernos del Centro de Estudios de Diseño y Comunicación. 10.18682/cdc.vi57.1459.

González, A. G. (2014). Efectos de la calidad de servicio y de la satisfacción del cliente sobre la fidelidad a los servicios oficiales de postventa de automoción españoles (Doctoral dissertation, Universidad de Alcalá).

Guillermo, Vázquez \& Mejía Trejo, Juan \& Núñez-Moreno, Tania. (2016). OPERACIONES $Y$ COMPETITIVIDAD EN LAS PYMES DEL SECTOR DEL PLÁSTICO EN LAS PYMES MANUFACTURERAS DE GUADALAJARA.

Kovalev, Yu.I.. (2021). The main principles of the strategic development of the industry. Vsyo o myase. 6-10. 10.21323/2071-2499-2021-46-10.

Marrero, S. M., \& Solari, E. (2015). Factores de las dificultades de financiación de las Pymes en 
Latinoamérica.

Ciencias administrativas, (6), 3-15.

Mejia Zambrano, J. P. (2019). ARTÍCULO CIENTÍFICO: Gestión de clientes basado en el modelo CRM para las Pymes del sector de la climatización de la ciudad de Guayaquil.

Morocho, F. R. A., \& Armas, C. S. B. (2017). Calidad en el Servicio: Oportunidad para el Sector Automotor en el Ecuador. INNOVA Research Journal, 2(9), 42-52.

Pereira Bolaños, Cliden. (2019). Actualidad de la gestión empresarial en las pymes. Apuntes Contables. 39-53. 10.18601/16577175.n24.03.

Raymond, Louis \& St-Pierre, Josée. (2018). The strategic development

of manufacturing SMEs-

Ycaza, D. E. M., \& Sánchez-Riofrío, A. M. (2016). Factores de competitividad orientados a la pequeña y mediana empresa (PYME) en Latinoamérica: revisión de la literatura. Revista San Gregorio, 2(15), 104-111.

Zribi, Hajer \& Zouaoui, Mahmoud. (2013). Strategic Development of SMEs in Developing Countries - Case of SOPAL-. Journal of North African Research in Business. Vol. 2013. 1. 10.5171/2013.848014. 